\title{
LA INCLUSIÓN DE LA HISTORIA DE LAS MUJERES EN EL NUEVO PARADIGMA EDUCATIVO DE LA HISTORIA:
}

\author{
ANÁLISIS CURRICULAR Y PROPUESTAS DIDÁCTICAS ${ }^{1}$
}

\author{
INCLUSÃO DE HISTÓRIA DE MULHERES NO NOVO PARADIGMA DE HISTÓRIA DA \\ EDUCAÇÃO: ANÁLISE E DE ENSINO PROPOSTAS CURRICULARES
}

\section{INCLUSION OF HISTORY OF WOMEN IN THE NEW PARADIGM OF EDUCATIONAL HISTORY: ANALYSIS AND TEACHING CURRICULUM PROPOSALS}

\author{
Carmen Suárez Suárez \\ Sonia García Galán²
}

\begin{abstract}
RESUMEM: Las "propuestas para el nuevo paradigma educativo de la historia" nos invitan a debatir sobre "el qué, el cómo y el porqué de la enseñanza de la historia en el nuevo siglo". La propuesta que presentamos, inscrita en este marco, pretende contribuir a dar respuesta a cuáles serían los aspectos básicos referidos a una educación pensada para el ejercicio de una ciudadanía corresponsable. Nuestra línea de reflexión pasa por la propuesta de una historia enseñada renovada que tenga en cuenta las investigaciones y los avances que se han producido en lo referido a la historia de las mujeres.
\end{abstract}

Palabras clave: Historia enseñada. Historia de las mujeres. Plan de estudios.

RESUMO: As "propostas para um novo paradigma da educativo da história" nos convidam a debater sobre "o que, como e porque do ensino de história no novo século". A propsota que apresentamos, se inscreve neste marco, pretendendo contribuir para responder aos aspectos básicos de uma educação pensada no exercício da cidadania responsável. Nesta linha, consideramos as invetigações sobre a história das mulleres.

Palavras-chabe: História ensinada. História das mulheres Currículo.

ABSTRACT: The "proposals for a new paradigm of educational history" invite us to discuss "what, how and why history of education in the new century." The propsota we present, falls within this framework and contributing to respond to the basic aspects of education thought in the exercise of responsible citizenship. Along these lines, we consider the invetigações on the history of mulleres.

Keywords: History taught. History of women. Curriculum.

\footnotetext{
1 Ver apresentação deste número da revista História \& Ensino, assim como a Proposta de um Novo Paradigma Educativo do grupo Historia a Debate (HaD) em: http://www.histedbr.fe.unicamp.br/revista/edicoes/28/art01 28.pdf

2 Universidad de Oviedo. Asturias, Espanha.
} 


\section{Introdución}

Las "propuestas para el nuevo paradigma educativo de la historia" nos invitan a debatir sobre "el qué, el cómo y el porqué de la enseñanza de la historia en el nuevo siglo" (BARROS, 2007, P. 1). ${ }^{3}$ La crisis económica actual, sus efectos sociales y las respuestas políticas ante la misma, inciden de lleno en un sistema educativo que es interpelado, al menos en el marco de los discursos, como un agente fundamental para la superación de los retos actuales. En el caso de España, se mira especialmente a los centros de enseñanza como parte esencial de la respuesta frente a la problemática del desempleo. Ante el imparable avance del conocido como New Paradigm in Education, resulta necesario elaborar una propuesta alternativa que supere la visión unívoca del proceso educativo como un espacio para la formación de mano de obra dúctil y adaptada a un mercado de trabajo volátil. Los retos de la educación han de pasar en el presente como en el futuro por la educación de nuevas generaciones capaces de mejorar, desde los principios emanados de los derechos humanos esenciales, la sociedad global que nos ha tocado vivir.

La propuesta que presentamos, inscrita en este marco, pretende contribuir a dar respuesta a cuáles serían los aspectos básicos referidos a una educación pensada para el ejercicio de una ciudadanía corresponsable. Cuestiones éstas, que deberían incluirse como contenidos para trabajar en las aulas de la Educación Secundaria y Bachillerato. Nuestra línea de reflexión pasa por la propuesta de una historia enseñada renovada que tenga en cuenta las investigaciones y los avances que se han producido en lo referido a la historia de las mujeres. Somos conscientes que aún no se ha configurado una historia que cuente con la presencia y la contribución de toda la humanidad pasada y presente, capaz de sintetizar y elevarse sobre europeísmos o marcos nacionales, pero sobre todo queremos poner de manifiesto que la historia que enseñamos en los niveles educativos medios debe superar el androcentrismo de la historiografía tradicional como elemento esencial para la consecución de una sociedad más justa y equitativa.

\footnotetext{
3 Barros, Carlos, "Propuestas para el nuevo paradigma educativo de la historia", p. 1. Disponible en: http://www.h-debate.com/cbarros/spanish/articulos/nuevo_paradigma/npeducativo.htm. [Consultada el 20 de agosto de 2014].
} 


\section{La educación por competencias: algunas perversiones y posibilidades}

Los altos niveles de paro ya referidos se han convertido en nuestro país en el aliado inesperado del New Paradigm in Education y de las teorías que sustentan una educación en la que prima el desarrollo de "competencias" o habilidades que preparen al alumnado para que sea capaz de adaptarse, en un futuro inmediato, a un precarizado mercado de trabajo. ${ }^{4}$ En el marco de la educación superior, las personas responsables de los Ministerios de Educación de Francia, Italia, Reino Unido y Alemania, establecían ya en la Declaración de la Sorbona -considerada como punto de arranque esencial para el proceso de creación de un Espacio Europeo de Educación Superior- una clara relación entre la precarización laboral y la renovación del sistema educativo al señalar que, ante la llegada de "un tiempo de cambios para las condiciones educativas y laborales" que implicaría la "diversificación del curso de las carreras profesionales" y habría de requerir "una formación continua" necesaria para dar respuesta a tales cambios, se hacía imprescindible generar un nuevo sistema de educación que ofreciese a los estudiantes oportunidades para buscar su ámbito de "excelencia". ${ }^{5}$ De esta forma, términos como "movilidad en el empleo" o "capacidad para la obtención de un empleo", citados en esta primera cumbre celebrada en 1998, han continuado formando parte de la agenda política europea en materia educativa en las reuniones posteriores (MONTERO CURIEL, 2010). Así, por ejemplo, la Declaración de Praga, en 2001, volvía a insistir en la importancia del aprendizaje a lo largo de toda la vida "para encarar los desafíos de la competitividad". ${ }^{6}$

\footnotetext{
${ }^{4}$ Las "competencias", hoy constituidas como elementos centrales del proceso de enseñanzaaprendizaje en el ámbito anglosajón y acogidas por sectores académicos latinoamericanos y administrativamente en Europa, proceden del campo de la psicología. Sus orígenes se relacionan con la publicación del conocido artículo de David McClelland quien, en 1973, cuestionaba las pruebas o test que medían la inteligencia y apostaba por otros sistemas de evaluación basados en habilidades o competencias. McClelland trataba de probar que la buena marcha en el ámbito académico no garantizaba el éxito profesional posterior ni en otras esferas de la vida, de lo que se infería que tenía más interés dotar al alumnado de esas habilidades para con ello mejorar sus posibilidades de empleo y promoción social. Las tesis de McClelland han sido, pese a su éxito aparente, discutidas por otros autores, que siguen estableciendo relaciones entre las habilidades cognitivas y el éxito profesional y personal. BARRET, Gerald V.; DEPINET, Robert L. (1991): A reconsideration of testing for competence rather than for intelligence. American Psycologist, n. 10, v.46, p. 1012-1024. 1991

5 "Declaración de Bolonia. Declaración conjunta de los Ministros Europeos de Educación", Bolonia, 19 de junio de 1999. Disponible en: http://www.eees.es/es/documentacion [Consultada el 20 de agosto de 2014].

6 "Declaración de Praga, 2001. Hacia el Área de la Educación Superior Europea. Declaración del encuentro de los Ministros Europeos en funciones de la Educación Superior en Praga, 19 de mayo
} 
Los mismos principios se han integrado en la legislación educativa española en los niveles medios. De esta forma, la Ley Orgánica de Educación de 3 de marzo de 2006 (LOE) establecía como principio director de la misma el "compromiso decidido con los objetivos educativos planteados por la Unión Europea" y, en concreto, "la pretensión de convertirse en la próxima década en una "economía basada en el conocimiento más competitiva y dinámica". ${ }^{7}$ En la misma línea, se incluía la intención de fomentar el "espíritu emprendedor", concepto éste desarrollado aún más en la más reciente Ley Orgánica de Mejora de la Calidad Educativa (LOMCE), que mantiene el compromiso de seguir con las directrices europeas en este campo. El Proyecto de Real Decreto por el que se establece el currículo básico de la educación primaria, de la educación secundaria obligatoria y del Bachillerato y que desarrolla los principios básicos recogidos por la LOMCE, establece de forma clara la decidida apuesta europeísta que deriva, a su vez, en una concepción del aprendizaje dirigido a la adquisición de "competencias":

En línea con la Recomendación 2006/962/EC del Parlamento Europeo y del Consejo de 18 de Diciembre de 2006 sobre Competencias Básicas para el aprendizaje a lo largo de la vida, toda la reforma educativa se basa en la potenciación del aprendizaje por competencias, como complemento al tradicional aprendizaje de contenidos. Se proponen nuevos enfoques en el aprendizaje y evaluación, que han de suponer un importante cambio en las tareas que han de resolver los alumnos y planteamientos metodológicos innovadores, no dirigidos a la cantidad de lo memorizado sino a aquello que el alumnado asimila y es capaz de hacer, sobre todo por lo que respecta a las competencias básicas: comunicación lingüística, y competencias en matemáticas, ciencia y tecnología e ingeniería, que se consideran prioritarias de cara al desarrollo del alumnado y a su capacidad de desenvolverse en el mundo del conocimiento y la tecnología. ${ }^{8}$

de 2001". Disponible en: http://www.eees.es/es/documentacion [Consultada el 20 de agosto de 2014].

7 Ley Orgánica de 2/2006, de 3 de mayo, de Educación. Disponible en:

http://noticias.juridicas.com/base datos/Admin/lo2-2006.html [Consultada el 20 de agosto de 2014].

8 El Proyecto de Real Decreto por el que se establece el currículo básico de la educación primaria, de la educación secundaria obligatoria y del Bachillerato conforme a la LOMCE establece claramente estos principios: "En línea con la Recomendación 2006/962/EC del Parlamento Europeo y del Consejo de 18 de Diciembre de 2006 sobre Competencias Básicas para el aprendizaje a lo largo de la vida, toda la reforma educativa se basa en la potenciación del aprendizaje por competencias, como complemento al tradicional aprendizaje de contenidos. Se proponen nuevos enfoques en el aprendizaje y evaluación, que han de suponer un importante cambio en las tareas que han de 
La noción de "competencias", entendidas como las aptitudes o capacidades que debería adquirir el alumnado en la educación primaria y en la secundaria obligatoria, con la intención de lograr aprendizajes útiles para la vida laboral, se convierte así en el eje central del proceso educativo. Las "competencias básicas" fueron establecidas por las distintas administraciones educativas en España a partir de la aprobación de la LOE. Aunque entre ellas se incluye una "competencia social y ciudadana" y otra competencia "cultural y artística" -denominadas ahora en LOMCE "competencias sociales y cívicas" y "conciencia y expresiones artísticas respectivamente ${ }^{-9}$ es innegable la importancia y prioridad concedida a aquellas habilidades que se consideran más funcionales y que son valoradas por el mercado laboral, tales como "aprender a aprender", el "tratamiento de la información y competencia digital" o la "autonomía e iniciativa personal", a las que la normativa desarrollada asociada a la LOMCE añade una más, el "sentido de iniciativa y espíritu emprendedor". ${ }^{10}$ Junto a estas capacidades cobran gran peso en los currículos, la denominada competencia "matemática" -a la que con la LOMCE se incorpora el subtítulo de "competencias básicas en ciencia y tecnología"- y la competencia en "comunicación lingüística", entendiendo estos saberes como esenciales por su carácter instrumental. Ésta última capacidad incluye, asimismo,

resolver los alumnos y planteamientos metodológicos innovadores, no dirigidos a la cantidad de lo memorizado sino a aquello que el alumnado asimila y es capaz de hacer, sobre todo por lo que respecta a las competencias básicas: comunicación lingüística, y competencias en matemáticas, ciencia y tecnología e ingeniería, que se consideran prioritarias de cara al desarrollo del alumnado y a su capacidad de desenvolverse en el mundo del conocimiento y la tecnología". Disponible en: http://www.mecd.gob.es/servicios-al-ciudadano-mecd/dms/mecd/servicios-al-ciudadanomecd/participacion-publica/cerrados/2013/curriculo-basico/RD-curr-culo-LOMCE-PR-ESO-BACHV1-10-12-13.pdf [Consultada el 20 de agosto de 2014].

9 Dado que todavía no se ha aprobado del currículo vigente para la educación secundaria, recogemos estas denominaciones conformes al Real Decreto que fija el currículo de primaria: "Real Decreto 126/2014, de 28 de febrero, por el que se establece el currículo básico de la Educación Primaria". Disponible en: http://www.boe.es/boe/dias/2014/03/01/pdfs/BOE-A-2014-2222.pdf [Consultada el 20 de agosto de 2014].

10 Algunas de las competencias más valoradas en el ámbito profesional son la capacidad comunicativa, la flexibilidad y la adaptabilidad al puesto -ligada a la noción de "aprender a aprender"- y la capacidad para resolver problemas y trabajar en equipo. CORRALES BERNABÉ, Damián. Estilos de aprendizaje de las competencias profesionales a lo largo de la vida. Estudio cualitativo en Cantabria. In: López, Fernando Guerra; GARCÍA-RUIZ, Rosa; FERNÁNDEZ Natalia González; ARELLANO Paula Renés; ZUBIZARRETA, Ana Castro (Coord.). Estilos de aprendizaje: investigaciones y experiencias. $V$ Congreso Mundial de Estilos de Aprendizaje. Santander, Universidad de Cantabria. 2012. Disponible en:

http://dialnet.unirioja.es/servlet/articulo?codigo $=4675074$ [Consultada el 20 de agosto de 2014]. Junto a esto, es innegable la importancia del manejo de las nuevas tecnologías y el aprendizaje de idiomas, especialmente en inglés, dada la globalidad de la economía y las interconexiones entre los mercados laborales nacionales e internacionales. 
el manejo de una o dos lenguas extranjeras. ${ }^{11}$ La educación por competencias constituye así el nuevo paradigma de la innovación en el campo educativo, simplificando a menudo los fines de un proceso tan complejo como esencial para el futuro de las sociedades, al limitar la educación a una herramienta instrumental para la búsqueda de un empleo y el crecimiento económico nacional. También se priman de forma evidente las disciplinas científico-técnicas sobre los saberes humanísticos, más desdibujados bajo principios generales en la legislación.

Esta nueva concepción del proceso educativo debe ser valorada en el sentido de que es innegable el papel de la educación obligatoria y post-obligatoria en la formación de la fuerza de trabajo. ${ }^{12}$ Junto a esto, cabe señalar la virtualidad de unos saberes que se transmiten y que se apropian por parte del alumnado de manera significativa, huyendo de un aprendizaje meramente memorístico y siendo capaces los alumnos y las alumnas de aplicar los contenidos a situaciones prácticas y cotidianas. Esta nueva perspectiva de enseñanza, que no ha de renegar de la explicación teórica magistral -cuando ésta es mesurada y parte de elementos tangibles para el alumnado- convierte a chicos y chicas en agentes activos de su propio proceso de aprendizaje, lo que contribuye a aumentar su motivación e interés por aprender y conocer. Asimismo, es cierto que la sociedad del conocimiento exige un manejo fluido y cotidiano de las nuevas tecnologías, obliga a una actualización continua -lo que se ha venido en llamar "aprender a aprender"y exige el manejo de una o más lenguas extranjeras. Sin embargo, el excesivo énfasis concedido por las administraciones y la legislación educativa a estas cuestiones instrumentales, vacía de contenidos el proceso de enseñanza. Sin ignorar las virtudes que puede aparejar una educación que tenga en cuenta el

\footnotetext{
${ }^{11}$ El interés de los gobiernos españoles por el aprendizaje de lenguas extranjeras y, particularmente el inglés, se traduce, en los últimos años en la proliferación de los programas de enseñanza "bilingües" que, pese a sus virtualidades, corren el peligro de priorizar la enseñanza y aprendizaje del idioma frente a los contenidos de la materia que se imparte en dicha lengua, teóricamente vehicular.

12 De hecho, y pese a los avances experimentados en los últimos años, los centros educativos son todavía receptores y emisores de roles de género que reproducen y refuerzan la división sexual del mercado de trabajo. En este sentido, la comunidad educativa debe mantener un esfuerzo continuo en aras de la igualdad de oportunidades real que garantice la libre elección de materias y ramas educativas y, en consecuencia, laborales. Corroboran estas aseveraciones los datos proporcionados por distintas encuestas y estudios realizados. Véase como ejemplo los datos sobre elección de ramas educativas y sobre el mercado de trabajo contenidos en el informe del Instituto Asturiano de la Mujer: "Mujeres y hombres en Asturias 2014". Disponible en:

http://institutoasturianodelamujer.com/iam/wp-content/uploads/2010/02/Diptico-Mujeres-yhombres-en-Asturias-2014.pdf [Consultada el 20 de agosto de 2014].
} 
desarrollo de competencias y habilidades, resulta necesario trasmitir y discutir sobre contenidos, y también sobre actitudes y valores.

La "competencia social y ciudadana" parecía destinada a acoger, en este sentido, aquellos aspectos formativos ligados con la igualdad y la crítica fundamentada ante las situaciones de injusticia o discriminación. Definida tras la aprobación de la LOE en el Real Decreto 1631/2006, de 29 de diciembre, por el que se establecían las enseñanzas mínimas correspondientes a la Educación Secundaria Obligatoria en toda España, esta competencia debía llevar al alumnado a comprender que "no toda posición personal es ética si no está basada en el respeto a principios y valores universales como los que encierra la Declaración de los Derechos Humanos". Junto a esto, la misma norma fijaba que había de implicar el reconocimiento de la igualdad de derechos entre hombres y mujeres y permitir, al mismo tiempo, reflexionar críticamente sobre conceptos como "democracia, libertad, igualdad, solidaridad, corresponsabilidad, participación y ciudadanía". ${ }^{13}$ La nueva formulación de esta competencia, tal y como se propone desde la aprobación de la LOMCE, desdibuja ya tales fines, pues no es lo mismo hablar de "civismo" que de "ciudadanía": el término "civismo" nos remite, según las acepciones recogidas por la RAE a "celo por las instituciones e intereses de la patria" $y$, en segundo lugar, a un "comportamiento respetuoso del ciudadano con las normas de convivencia pública", ${ }^{14}$ mientras que la noción de ciudadanía se vincula, como es bien sabido, a derechos políticos y a su ejercicio. La primera llama a la quietud y al ardor patriótico, la segunda al ejercicio responsable de derechos y obligaciones y a la protesta si cabe, ante el incumplimiento de los mismos. Junto a esto, cabe señalar la eliminación por parte de la LOMCE de la materia "Educación para la ciudadanía", creada en España por la LOE en consonancia con asignaturas similares existentes en otros países europeos. Dicha materia tenía sus carencias, pues estaba diseñada sobre todo para mostrar el sistema político nacional y europeo y para hacer partícipe al alumnado del mismo, pero sin llegar a cuestionar de forma crítica los límites, deficiencias o fisuras que persisten en el

\footnotetext{
13 Real Decreto 1631/2006, de 29 de diciembre, por el que se establecen las enseñanzas mínimas correspondientes a la Educación Secundaria Obligatoria. Disponible en:

http://www.boe.es/boe/dias/2007/01/05/pdfs/A00677-00773.pdf [Consultada en 20 de agosto de 2014].

14 Diccionario de la Lengua Española. Real Academia de la Lengua. Disponible en: http://lema.rae.es/drae/?val=civismo
} 
funcionamiento de las democracias. Con todo, compensaba en cierta medida el déficit al que se somete la formación humanística en un sistema educativo que privilegia, como ya se ha visto, las competencias instrumentales y técnicas.

Aunque mermadas en su formulación por la LOMCE, "las competencias sociales y cívicas" deberían contribuir a que los alumnos y las alumnas puedan ejercer ya en su juventud y sobre todo en su madurez, una ciudadanía responsable, crítica e informada. El cumplimiento de los derechos humanos, la superación de las desigualdades de toda índole y el desafío ecológico al que nos enfrentamos requieren jóvenes con capacidad para buscar y contrastar información, pero también con unos conocimientos básicos que les permitan situarse en el contexto global actual. Que seamos capaces de favorecer que nuestro alumnado adquiera esa competencia pasa por revisar el modo de enseñar cómo se ha construido históricamente la ciudadanía. Pasa también porque seamos conscientes de que la desigualdad entre mujeres y hombres es un producto cultural e histórico, que persiste en el mundo actual con diferentes manifestaciones dependiendo del espacio geográfico que estemos considerando. Desde la materia Ciencias Sociales, Geografía e Historia, podemos y debemos contribuir a que el alumnado tome conciencia de estas problemáticas, porque solo desde el conocimiento se podrá adquirir una actitud crítica necesaria para el cambio social. La visibilidad de las mujeres en la historia, tanto de su situación de subalteridad pasada y presente, como de su contribución al avance de las sociedades resulta esencial para generar preguntas y debates y para generar cambios y modificar conciencias marcadas por los estereotipos y roles de género. Nos proponemos realizar, en el siguiente apartado, una crítica a los contenidos de los currículos en materia de historia de las mujeres, para con ello visualizar los déficits heredados y persistentes sobre los que construir una propuesta alternativa.

\section{De la LGE a la LOMCE}

\subsection{Primeros intentos renovadores}

En los últimos treinta años el sistema educativo español ha estado sometido a un conjunto de disposiciones normativas a los efectos de democratizar los 
principios "tecnocráticos" en los que se había inspirado la Ley 14/1970 General de Educación del Franquismo. La transformación de esta ley en el periodo democrático, después de 1975, experimentó un largo recorrido que supuso las promulgaciones, en primer lugar, de la Ley Orgánica 8/1985 reguladora del Derecho a la Educación (LODE) que daba satisfacción a los principios recogidos en el artículo 27 de la Constitución Española de 1978 respecto a la libertad de creación de centros $\mathrm{y}$, por tanto, al establecimiento de una doble red pública y privada y, en segundo lugar, de la Ley 1/1990 de Ordenación General del Sistema Educativo (LOGSE) que vino a consolidar una primera "democratización" abierta a las nuevas exigencias del contexto europeo, al que ya pertenecíamos.

En ese periodo citado anteriormente de, al menos quince años antes de la entrada en vigor de la LOGSE, se acentuaron las reflexiones sobre cómo debía ser una propuesta razonable para un cambio en la educación. A la vez que esto sucedía, se publicaban las primeras investigaciones sobre la historia de las mujeres en nuestro país abriéndose así camino una primera visión del pasado en femenino que se centraba sobre todo en las heroínas y la "emancipación". Aquellas aproximaciones tuvieron como referencia la Segunda República y la Guerra Civil, un espacio privilegiado para hacer visibles a las españolas, en el que habían conquistado derechos civiles y políticos negados durante el período liberal (RAMOS PALOMO, 2006, p. 22). Éstas fueron las aportaciones de pioneras como Mary Nash, Rosa María Capel Martínez y Amparo Moreno, hispanistas como Giuliana di Febo y escritoras como Aurelia Capmany, Carmen Alcalde y Teresa Pamies, entre otras. Al mismo tiempo, se iniciaba en España la reflexión teórica que en buena medida partía de la historiografía francesa y la anglosajona. Así categorías como "género" permitieron, ya en los años 90 del siglo XX, establecer el modelo de relaciones entre los sexos y categorizar muchos conceptos de la nueva historia de las mujeres y de género (androcentrismo, patriarcado, contrato sexual) y revisar otros (familia, reproducción, identidad, poder, clase, ciudadanía) (NASH, 1991; RAMOS PALOMO, 2003; DEL VAL VALDIVIESO, 2004).

De forma paralela, en la década de 1980, un conjunto de profesores y profesoras se integraron en movimientos de renovación pedagógica planteando muchas propuestas alternativas para una docencia que en tiempos de transición resultaba ya obsoleta. Las importantes reflexiones que se produjeron en ellos 
dejaron una secuela profunda porque acompañaban a las innovaciones didácticas en la práctica, una reflexión teórica sobre el papel del profesorado y una fuente constante de reconocimiento y autocrítica de su labor, siempre atenta a la mejora docente. Sus reflexiones sobre el Libro Blanco para la Reforma del Sistema Educativo (1989) fueron tan productivas e interesantes que las Administraciones Educativas utilizaron su bagaje teórico-práctico como importante fuente de información para algunas de sus decisiones políticas (ROGERO ANAYA, 2010).

Una parte de esta aportación llevaba aparejada, además, una reflexión sobre el androcentrismo y el sexismo en todos los ámbitos de las instituciones escolares incluida la docencia en los centros educativos de la educación obligatoria. Estas ideas y debates, propiciados sobre todo por algunas profesoras, se concretaron en ámbitos de formación llamada "transversal". La "Educación para la Igualdad de Oportunidades de ambos sexos" como contenido transversal se esperaba "impregnara" las áreas curriculares (MAÑERU MÉNDEZ; HERRÁEZ, 1992; SEBASTIÁN RAMOS; LIÉVANO; SÁNCHEZ GARCÍA, 2001). Una de las estrategias más importantes afrontadas por las Administraciones Educativas entonces consistió en generar toda una red de responsables de formación y recursos en el conjunto del territorio español. En este sentido, dos cursos de postgrado que se celebraron en la Universidad de Barcelona en los años 1990 y 1991 fueron dirigidos por la catedrática de Historia Contemporánea de la Universidad de Barcelona Mary Nash y contaron con la subvención y el patrocinio del Ministerio de Educación y Ciencia. Tuvieron una orientación interdisciplinar $y$, en los mismos, prestigiosas profesoras ejemplificaron en sus ponencias las últimas investigaciones sobre los estudios de las mujeres en España y en Europa. Ida Blom de la Universidad de Bergen (Noruega), que en la primera edición inauguró el curso, disertó sobre "El género como categoría de análisis: un enfoque interdisciplinar"; Sandra Acker, profesora de la Universidad de Bristol (Gran Bretaña) planteó el "Análisis de género en el sistema educativo británico"; Maria Grever, de la Universidad Erasmus de Van Nijmegen (Países Bajos), expuso la "Historia de las mujeres en la Educación Secundaria: la experiencia holandesa" y Karin Hause del Institut für 
Geschichwissenschaft de la Universidad Técnica de Berlín abordó "El género como categoría de análisis, Un enfoque interdisciplinar", entre otras. ${ }^{15}$

La denuncia sobre las raíces del androcentrismo y la visión patriarcal del mundo y de la ciencia comenzó a ser divulgada desde las aulas universitarias y desde las aulas de las enseñanzas medias. En Liberación y utopía, obra dirigida por María Ángeles Durán, Cristiana Segura abordaba "La incidencia de la mujer en la ciencia histórica", señalando el camino recorrido por ella misma como profesional. Se consideraba consciente de su educación en el androcentrismo, su participación en él, pero vislumbraba la posibilidad de escribir una historia de las mujeres (GRAÍÑ̃, 1982). Aún formadas en el discurso androcéntrico, las historiadoras españolas se hicieron conscientes de poder transformador que tenía la docencia, la investigación, y en este sentido, rescatar a las mujeres para la historia. En lo que atañe al segundo aspecto, investigadoras como Amparo Moreno analizaron con detalle los contenidos que se transmitían en los libros de texto en las enseñanzas medias. En este punto, El arquetipo viril protagonista de la historia. Ejercicios de lectura no androcéntrica, señala pautas muy precisas sobre el peso de la transmisión de una ideología patriarcal y la necesidad de ofrecer lecturas más igualitarias que cuenten con una presencia equilibrada de mujeres y hombres. Moreno señala además que considerar como "natural" unas relaciones no igualitarias del poder llevaba a asimilar éstas a la práctica de la vida diaria (Moreno, 1986, p. 109). Los libros de texto, aún anclados en el pasado, seguían reproduciendo viejos esquemas de la historia franquista y eran ajenos a las nuevas contribuciones de las investigaciones académicas. Algunos textos rompían esta tradición como el manual de Esmeralda González, José María de Juana y José Ortega para el Bachillerato Unificado y Polivalente. Este libro del curso tercero de Historia de las Enseñanzas Medias incluía un novedoso enfoque teórico en forma de grandes esquemas interpretativos y unas propuestas de trabajo prácticas para el alumnado basadas en la reflexión sobre fuentes y documentos históricos relevantes. Pero no había, no obstante, ninguna referencia sobre las mujeres, su

15 I Curso de Postgrado para responsables de coeducación. Ministerio de Educación y Ciencia. Universidad de Barcelona. Centro de Investigación Histórica de la Mujer. Barcelona, febrero-junio de 1990; II Curso de Postgrado para responsables de coeducación. Ministerio de Educación y Ciencia. Universidad de Barcelona. Centro de Investigación Histórica de la Mujer. Barcelona, enero-mayo de 1991. 
historia y su protagonismo. ${ }^{16}$ Por ello, multitud de propuestas pedagógicas innovadoras se plasmaron en materiales para el trabajo de aula como alternativas al libro de texto y así fue como se solventaron en la práctica las ausencias y silencios sobre las mujeres.

Esta trayectoria docente y pedagógica con pretensiones innovadoras se plasmó en parte en la publicación de la LOGSE y posteriormente de la Ley Orgánica 9/1995 de Participación, Evaluación y Gobierno de los Centros (LOPEGCE). El difícil proceso de implantación de la LOGSE se dilató en el tiempo y derivó, en muchas ocasiones, en una pérdida de sentido de los principios básicos en los que se había basado, que establecían, entre otras cuestiones, que en sociedad del futuro configurada progresivamente como una sociedad del saber, la educación compartirá con otras instancias sociales la transmisión de información y conocimientos, pero adquirirá aún mayor relevancia su capacidad para ordenarlos críticamente, para darles un sentido personal y moral, para generar actitudes y hábitos individuales y colectivos, para desarrollar aptitudes, para preservar en su esencia, adaptándolos a las situaciones emergentes, los valores con los que nos identificamos individual y colectivamente. ${ }^{17}$

Podría esperarse que con un marco legal abierto hacia la crítica y la innovación docente, como el que iniciaba la LOGSE, a través por ejemplo de las propuestas de formación, con las que se crearon redes de docentes implicadas en tareas coeducativas e igualitarias, se avanzaría hacia una educación más justa y asimismo en el conocimiento de una historia "mixta" y compensada de protagonismos y presencias. Con estas bases, se podría haber esperado que las actitudes del profesorado cambiaran y que los niveles de conciencia social del alumnado se incrementaran. Pero en la práctica los logros conseguidos en este sentido fueron más modestos, si bien, podemos señalar, que se inició una nueva vía en esta dirección.

Desde los comienzos del nuevo milenio, la historiografía de las mujeres en España, ya consolidada como área de investigación en el terreno universitario,

16 URRUELA Esmeralda González; GONZÁLEZ, José María de Juana; VALCÁRCEL, José Ortega España. Geografía e Historia. Santiago Rodríguez Hermanos: Burgos. 1978. Este manual siguió vigente hasta finales de los años ochenta del siglo XX.

17 LOGSE. Ley 1/1990 de 3 de octubre (BOE de 4 de octubre de 1990). Disponible en: http://www.educacion.gob.es/mecd/oposiciones/files/logse.pdf [Consultado el 21 de agosto de 2014]. 
avanza en el conocimiento del pasado e incluye nuevas áreas temáticas y metodológicas (RAMOS PALOMO, 2006, p.14). La necesidad de reconstruir una historia general que cuente con la voz y la experiencia de las mujeres es cada vez más evidente y visible. Aunque recibe el empuje de las nuevas corrientes posmodernas que llevan a cuestionar el objetivo clásico de sacar a la luz una historia de las mujeres "propia" que a su vez sea capaz de favorecer la reescritura de una historia general que tengan en cuenta a hombres y mujeres históricamente y abogan, por el contrario, por una historia de género más centrada en los discursos, significados y en las relaciones de poder, en líneas generales, esta corriente mantiene en el caso español su vinculación con una historia social renovada (BORDERÍAS, 2006). La precariedad de las universidades y de la investigación en España en la actualidad, más acentuada aún en el campo de las humanidades, hace difícil vislumbrar un relevo generacional necesario que garantice el mantenimiento de la historia de las mujeres como la corriente renovadora que ha sido en los últimos años. ${ }^{18}$ Pese a ello, el acervo de conocimientos acumulado permite ofrecer visiones generales de las distintas épocas y sociedades que deberían incorporarse a los currículos en la enseñanza primaria como, sobre todo, en la enseñanza secundaria y en el bachillerato. La LOE, aprobada en 2006, constituyó, un primer intento por transmitir una "historia equilibrada de mujeres y hombres". No obstante, pese a sus buenas intenciones en este sentido, presentó, como veremos en el siguiente apartado, claros déficits.

\subsection{La Ley 2/2006 Orgánica de Educación (LOE): hacia una historia mixta. ${ }^{19}$}

La aprobación de la LOE vino precedida por la Ley Orgánica 1/2004 de 26 de diciembre, de Medidas de Protección integral contra la Violencia de Género en cuyo contenido se recogía la importancia de los procesos de socialización y, en concreto, cómo en el ámbito educativo debía fomentarse "la eliminación de los obstáculos que dificultan la plena igualdad entre hombres y mujeres" y cómo las

\footnotetext{
18 El manifiesto de Historia a Debate señala en su punto XII la importancia de un relevo generacional que continúe apostando por una historia renovada, socialmente útil y sustentada en una autocrítica constante. "Manifiesto de Historia a Debate", Pasado y Presente. Revista de Historia, v.8, n.15, p.168-183.

19 Esta ley vino precedida de otra non nata Ley 10/ 2002 Orgánica de Calidad de la Educación (LOCE).
} 
Administraciones Educativas debían velar "para que en todos los materiales educativos se eliminen los estereotipos sexistas o discriminatorios". Cabe señalar además que tuvo su continuidad en la Ley Orgánica 3/2007, de 22 de marzo, para la igualdad efectiva de mujeres y hombres que establecía en su art. 24. f) "El establecimiento de medidas educativas destinadas al reconocimiento y enseñanza del papel de las mujeres en la Historia", incidiendo además en la necesaria conexión entre las enseñanzas obligatorias y básicas y las superiores respecto al principio de equidad en la educación. Finalmente la Ley Orgánica 2/2010, de 3 de marzo, de salud sexual y reproductiva y de la interrupción voluntaria del embarazo estipuló que la salud sexual y reproductiva formaría parte de la educación del alumnado "como parte del desarrollo integral de la personalidad y de la formación en valores" (art. 9).

La aprobación de estas normas servía como punto de apoyo para un cambio en el proceso de enseñanza de la historia desde la perspectiva de la igualdad de oportunidades entre mujeres y hombres. No obstante, como tuvimos ocasión de señalar en el IV Congreso Internacional Historia a Debate, celebrado en Santiago de Compostela en 2010, el currículo vigente en Educación Secundaria acorde con la LOE, adolecía de omisiones y perversiones en este campo. A modo de ejemplo analizamos los currículos en vigor del Principado de Asturias aprobados en 2007 y 2008 para las materias de Ciencias Sociales y para la Historia del Mundo Contemporáneo e Historia de España. ${ }^{20}$ A pesar de contener la declaración expresa de la necesidad de reflejar "la participación de las mujeres en el ámbito público, con el fin de evidenciar que la historia de la humanidad es el resultado de la contribución de todas las personas, tanto hombres como mujeres", los silencios relativos a las féminas eran constantes: en los primeros años de la Educación Secundaria Obligatoria ( $1^{\circ}$ y $2^{\circ}$ curso) no había ninguna referencia a ellas. En el tercer cuso, dedicado al estudio de la Geografía, se hacía una alusión al "género" como una categoría válida al tratar el desarrollo humano, la pobreza y su

20 Decreto 74/2007, de 14 de junio, por el que se regula la ordenación y establece el currículo de la Educación secundaria obligatoria en el Principado de Asturias. Disponible en:

http://www.educastur.es/index.php?option=com content\&task=view\&id=997\&Itemid=152 [Consultado el 27 de octubre de 2010].

Decreto 75/2008, 6 de agosto, por el que se establece la ordenación y el currículo del Bachillerato. Disponible en:

http://www.educastur.es/index.php?option=com content\&task=view\&id=2060\&Itemid=54

[Consultado el 27 de octubre de 2010]. 
feminización. En cuarto curso, consagrado al estudio de la historia contemporánea, las mujeres aparecían exclusivamente vinculadas al movimiento sufragista. En el bachillerato, la materia optativa Historia del Mundo Contemporáneo volvía a incidir en los contenidos al sufragismo ya aludido y de nuevo a la feminización de la pobreza, y se incluía una referencia al feminismo como parte de los nuevos movimientos sociales. En la materia Historia de España, obligatoria para todo el alumnado en segundo de bachillerato, se limitaban a incluir dos alusiones: la cuestión del voto como consolidación de derechos políticos y sociales en la Segunda República y la "progresiva emancipación social y económica de las mujeres" en la España democrática.

Las omisiones de la norma se traducían, perpetuaban o agravaban, según los casos, en los libros de texto, que reflejaban visiones estereotipadas sobre las mujeres y los varones. ${ }^{21}$ En los contenidos de Prehistoria, por ejemplo, se presentaba a las mujeres tejiendo, tratando pieles, moliendo el grano, haciendo cestería o alfarería y, en todo momento, cuidando a sus criaturas; mientras los varones cazaban, construían, fundían minerales y portaban armas. En la Edad Antigua se señalaba, sin profundizar más, que las mujeres permanecían bajo la tutela del pater familias, pero en ningún momento se preguntaban cuestiones tales como si era importante el trabajo de las mujeres, cuando sabemos que ejercieron oficios diversos como tintoreras, nodrizas, maestras, taberneras, artesanas y vendedoras, entre otros. En el campo de la Geografía, los textos abordaban la problemática de la población mundial y las tasas demográficas, situando el debate en el papel de los Estados y en las determinaciones que adoptan respecto, pero en ningún caso se analizaban las condiciones demográficas específicas de las mujeres, la situación derivada del ejercicio de la maternidad, la tipología familiar que condiciona la vida de las mujeres, los desequilibrios producidos por la pobreza que es, sobre todo, femenina. Se obviaban igualmente las condiciones generales de salud sexual y reproductiva en los distintos contextos nacionales, políticos y sociales que facilitarían una cartografía de las poblaciones distinta a las ofrecidas por los materiales didácticos. Por último, en lo referente a la historia de

\footnotetext{
21 Los manuales más utilizados en las aulas eran los editados por ANAYA; SANTILLANA; BURGOS ALONSO, Manuel; MUÑOZ-DELGADO, María Concepción. Ciencias Sociales. Geografía e Historia. Madrid: Anaya, 2008; GRENCE RUIZ, T. (Dir.). Ciencias Sociales. Geografía e Historia. Madrid: Santillana. 2008.
} 
España, la aprobación del voto femenino se presentaba de manera aislada, obviando conceptos básicos como la propia noción de ciudadanía y la exclusión de las mujeres y otros colectivos de la misma.

Pese a estas carencias, la LOE representó, como ya apuntamos, un avance de cara a la inclusión de la historia de las mujeres en las aulas de los institutos y colegios españoles. Aunque la norma de por sí ya contenía carencias significativas, agravadas por los materiales de uso más común en el trabajo docente, los libros de texto, abría la posibilidad a nuevas perspectivas, contenidos, análisis y valores sobre la máxima de lograr una enseñanza de la historia más equilibrada que revirtiera a su vez en la tarea de combatir la discriminación por razón de género y modificase, en un plazo más amplio, la posición subalterna de las mujeres en la sociedad. Las virtudes de la LOE son aún más visibles cuando la comparamos con la LOMCE, auténtica contrarreforma educativa que desdibuja los fines apuntados y retrotrae al sistema educativo español a un proceso de reválidas y selección de los "mejores" en la búsqueda de la "excelencia".

\subsection{La LOMCE: una contrarreforma educativa}

Las nuevas disposiciones normativas, el marco señalado por la LOMCE y las primeras propuestas de Reales Decretos para la Educación Secundaria y el Bachillerato establecen un nuevo contexto para la enseñanza de la historia y, en concreto, de historia de las mujeres, que es necesario analizar. ${ }^{22}$

En primer lugar, resulta clave valorar qué contempla en lo referido a la presencia de los principios de igualdad el "Proyecto de real decreto por el que se establece el currículo básico de la educación primaria, de la educación secundaria obligatoria y del bachillerato". ${ }^{23}$ En principio, cabe señalar que el artículo 6.3 de este proyecto establece que "las Administraciones educativas fomentarán el desarrollo de los valores que fomenten la igualdad efectiva entre hombres y

22 Se ha publicado el Real Decreto 126/2014, de 28 de febrero, por el que se establece el currículo básico de la Educación Primaria (BOE del 1 de marzo) así como los proyectos de Reales Decretos por los que se establece el currículo de la Educación Secundaria y el Bachillerato.

23 Disponible en:

http://www.mecd.gob.es/servicios-al-ciudadano-mecd/participacion-

publica/cerrados/2013/curriculo-basico.html (consultada 11-5-2104). Ha sido publicado el 10-122013 en la web del Ministerio de Educación. 
mujeres, así como la prevención de la violencia de género", inscribiéndose en el marco de la Ley 1/2004, de 28 de diciembre, de Medidas de Protección Integral contra la Violencia de Género así como en la Ley orgánica 3/2007, de 22 de marzo, para la igualdad efectiva de mujeres y hombres. Además, explicita que la educación secundaria obligatoria tendrá entre sus fines y entre sus objetivos (art. 19):

- Valorar y respetar la diferencia de sexos y la igualdad de derechos y oportunidades entre ellos. Rechazar los estereotipos que supongan discriminación entre hombres y mujeres.

- Fortalecer sus capacidades afectivas en todos los ámbitos de la personalidad y en sus relaciones con los demás, así como rechazar la violencia, los prejuicios de cualquier tipo, los comportamientos sexistas y resolver pacíficamente los conflictos

- Conocer y aceptar el funcionamiento del propio cuerpo y el de los otros, respetar las diferencias, afianzar los hábitos de cuidado y salud corporales e incorporar la educación física y la práctica del deporte para favorecer el desarrollo personal y social. Conocer y valorar la dimensión humana de la sexualidad en toda su diversidad. Valorar críticamente los hábitos sociales relacionados con la salud, el consumo, el cuidado de los seres vivos y el medio ambiente, contribuyendo a su conservación y mejora.

De igual manera para el Bachillerato, se concretan fines y objetivos semejantes (art. 29):

- Ejercer la ciudadanía democrática, desde una perspectiva global, y adquirir una conciencia cívica responsable, inspirada por los valores de la Constitución española así como por los derechos humanos, que fomente la corresponsabilidad en la construcción de una sociedad justa y equitativa.

- Fomentar la igualdad efectiva de derechos y oportunidades entre hombres y mujeres, analizar y valorar críticamente las desigualdades 
existentes e impulsar la igualdad real y la no discriminación de las personas con discapacidad.

- Conocer y valorar críticamente las realidades del mundo contemporáneo, sus antecedentes históricos y los principales factores de su evolución. Participar de forma solidaria en el desarrollo y mejora de su entorno social.

Si se reconoce que las Ciencias Sociales en la educación secundaria obligatoria han de contribuir al "conocimiento de la sociedad, su organización y funcionamiento a lo largo del tiempo" como elemento "esencial para poder entender el mundo actual y, de alguna forma, realizar una previsión del futuro", y si, además, se reconoce que han de tener un importante papel en "la adquisición de la competencia social y ciudadana, en cuanto permiten la comprensión de la realidad social, actual e histórica", no es posible que estén ausentes las mujeres de los contenidos de esta materia obligatoria para todo el Estado y que formarán parte de la evaluación de los estándares. Y es que la LOMCE establece como elemento clave los llamados "estándares de aprendizaje evaluables", cuya referencia son los criterios de evaluación pero que, y esto es lo más importante, serán traducidos a pruebas estatales homologables y soporte para la obtención de los títulos de Secundaria Obligatoria y de Bachillerato.

En estos estándares, dictados por el Ministerio de Educación, la historia de las mujeres está ausente. Un análisis detallado de los escenarios, o sea los contenidos de aprendizaje del alumnado, muestra la ausencia total de referencia a la presencia de las mujeres en el relato histórico. Las distintas Comunidades Autónomas, en el uso de sus competencias, podrán aportar algunos aspectos del currículo pero sus propuestas de Decretos aún no están publicadas por lo que es, de momento, imposible analizar si contemplarán las carencias en este sentido. ${ }^{24}$ Lo que se perfila, no obstante, es la falta de interés en que la historia de las mujeres forme parte de la formación cívica básica que se pretende alcance todo el alumnado al término de su etapa obligatoria y post-obligatoria.

\footnotetext{
${ }^{24}$ En este nuevo marco las administraciones educativas podrán complementar los contenidos del bloque de asignaturas troncales, específicas y de libre configuración autonómica, realizar recomendaciones metodológicas así como fijar el horario lectivo máximo.
} 
Y redundando más, si la Historia de España "deberá contribuir a fomentar una especial sensibilidad hacia los problemas del presente, que anime a adoptar una actitud responsable y solidaria con la defensa de la libertad, los derechos humanos y los valores democráticos" y si la Historia del Mundo Contemporáneo "debe acercar al alumnado al conocimiento del mundo actual, y presentar la Historia como un proceso en el que inciden todos los aspectos de la vida del hombre", no entendemos cómo cabe omitir la subalteridad femenina en la explicación de las sociedades del pasado y del presente. Pensamos, en definitiva, que no es posible una educación cívica, responsable y democrática olvidando las acciones y presencia desigual de la mitad de la humanidad.

Cabe destacar, por tanto, esta omisión u ocultación en los currículos que, dado el marco actual, quizás solo pueda paliarse mediante una respuesta "desde abajo", a través de la formación continua del profesorado y la creación de redes entre la Universidad y la enseñanza secundaria, como medio para incentivar una renovación pedagógica crítica y la puesta en práctica de un paradigma educativo de la historia renovado e integrador, que corrija o palie los déficits de la legislación educativa.

\section{La historia social de las mujeres y el nuevo paradigma educativo de la historia: de los consensos a la creación de redes y grupos de trabajo}

En consonancia con las "Propuestas para el nuevo paradigma educativo de la historia", coincidimos en la importancia de pensar y debatir sobre cómo se ha escrito la historia y, más aún en el caso que nos ocupa, sobre cómo la estamos trasmitiendo. En los últimos tiempos parece que toda innovación educativa proviene de quienes abogan por un sistema educativo más eficiente en términos de formación de mano de obra $y$, en línea con esta tendencia se habla de "competencias", "calidad" o "fomento del espíritu emprendedor". Desde nuestra óptica, la innovación educativa debe partir, como en las experiencias de los años 80 , de la reflexión y el trabajo de la propia comunidad docente, que debe actualizar sus conocimientos -adquiridos para después trasmitirlos- a través del contacto con la comunidad investigadora y universitaria. Quienes trabajan a diario en las aulas, 
los profesores y las profesoras, son los verdaderos instrumentos para el cambio educativo (GRAU COMPANY; GÓMEZ LUCAS; PERANDONES GONZÁLEZ, s/d)). EI nuevo paradigma educativo de la historia debe contar con ellos y con ellas, abogando por una enseñanza que sin renunciar a las capacidades y a la calidad apueste decididamente por la crítica, la equidad y la mejora de las sociedades.

El conocimiento histórico porta en sí mismo un mensaje revolucionario, pues las desigualdades y logros del pasado nos permiten vislumbrar más claramente el presente sin renunciar a mejorar el futuro. La historia de las mujeres contribuye a estos fines generales al desvelar el desigual papel de hombres y mujeres a lo largo de la historia y poner de manifiesto, en el espejo del pasado, los retos actuales que distan para lograr una verdadera igualdad de oportunidades. La historiografía de las mujeres y el género ha aportado nuevas fuentes y nuevas lecturas de fuentes tradicionales que han de ser tenidas en cuenta: las cartas, los diarios, las fotografías, los testimonios orales, entre otras, son ventanas que nos permiten acercarnos a un pasado que no resulta tan distante, frío o indiferente para el alumnado. Poner rostro a la gente común o excepcional de las sociedades del pasado, contribuye a despertar el interés por el conocimiento histórico.

En un mundo cada vez más global e interconectado, es importante trasmitir al alumnado las relaciones e interacciones que se establecen entre las distintas regiones del planeta y entender que todos y todas constituimos sujetos de la historia. El feminismo postcolonial aporta interesantes reflexiones para la construcción de una globalidad más incluyente, de las que se ha servido la historia de las mujeres: situado el varón blanco occidental como sujeto central de la historia, el relato tradicional ha omitido a las otras, las mujeres, pero también a otras etnias y pueblos colonizados (FEMENÍAS, 2013; BERBEK SÁNCHEZ; JIMÉNEZ; MOSQUERA. 2013).

Éstas y otras aportaciones de la historia de las mujeres y del género y, en suma, una visión historiográfica social y renovada, puede transmitirse al profesorado de los institutos e incluso de los colegios, a través de cursos de formación continua. Desde el Grupo Deméter. Historia, mujeres y género de la Universidad de Oviedo, dirigido por la profesora Rosa María Cid López, llevamos 
varios años trabajando en esta línea. ${ }^{25}$ Como grupo de investigación ligado al ámbito académico, pensamos que los y las jóvenes de los institutos de enseñanza secundaria tienen que recibir el caudal de conocimientos aportado por las investigaciones en el campo de la historia de las mujeres y el género. La colaboración del profesorado de estos centros educativos resulta crucial para poner sobre la mesa las posibilidades y las dificultades de llevar adelante el proyecto. Desde su experiencia cotidiana, los y las docentes pueden ayudarnos a reflexionar sobre qué historia estamos transmitiendo, qué contenidos y valores diferentes queremos incluir, y cómo modificar los patrones de conducta heredados. Al mismo tiempo, su experiencia resulta crucial para evidenciar las dificultades que cabe encontrar en este intento de renovación y cambio pedagógico, derivadas por ejemplo de unos currículos con graves omisiones y al mismo tiempo recargados de contenidos pormenorizados, vinculados a una concepción de la historia positivista ya superada.

Estas intenciones y reflexiones nos llevaron a colaborar con los Centros del Profesorado y Recursos (CPR) que son las entidades que, como parte de los Planes de Formación, ofrecen la posibilidad de organizar cursos específicamente dirigidos a propiciar un nuevo enfoque en la enseñanza de la historia. Plantear esta propuesta en el marco de los CPRs presenta múltiples ventajas: en primer lugar, las profesoras y los profesores están familiarizados con la oferta de estos organismos. En segundo lugar, los cursos que realizan en el seno de los mismos contabilizan para la carrera profesional y la mejora retributiva del personal funcionario, lo que supone una motivación extra para la matrícula en los mismos. En tercer lugar, las sedes que se utilizan para su desarrollo están bien dotadas de medios tecnológicos y repartidos por la geografía regional. En nuestro caso, nuestra oferta se ciñe al Principado de Asturias, que cuenta en con cuatro centros de estas características: Avilés-Occidente, Oviedo, Cuencas Mineras y GijónOriente. Finalmente, plantear nuestra propuesta formativa desde estos espacios nos permite disponer del apoyo de una persona de referencia, trabajadora del CPR,

\footnotetext{
${ }^{25}$ El Grupo de investigación Deméter. Historia, mujeres y género se constituyó en 2007 en el seno de la Universidad de Oviedo. Actualmente es un "grupo de investigación de excelencia" especialista en historia de las mujeres y estudios de género integrado fundamentalmente por historiadoras pero con aportaciones procedentes de juristas.
} 
que conoce el perfil del profesorado destinatario y colabora en la planificación, desarrollo y evaluación de las actividades.

Los cursos se estructuran en dos tipos de sesiones: las primeras, de contenido más teórico, incluyendo el manejo de fuentes y documentos de diversa índole, y las segundas, de tipo práctico, dedicadas a trabajar sobre cómo trasladar los contenidos vistos a la actividad diaria en el aula. Este tipo de sesiones prácticas tipo "taller" tienen además la virtualidad de favorecer la buena marcha dinámica de grupo, propiciando el establecimiento de relaciones personales y profesionales entre el profesorado asistente. Este aspecto no debe descuidarse puesto que de estas relaciones interpersonales pueden surgir grupos de trabajo que en el medio plazo generen estrategias de innovación educativa que favorezcan la inclusión de la historia de las mujeres en las aulas.

Hemos planificado y llevado a cabo tres cursos de contenidos diferentes, siendo éstos pioneros en ocuparse de la trasmisión de la historia de las mujeres y el género en los Centros de Profesores y Recursos del Principado de Asturias. El primero de ellos tuvo lugar en el año 2012 en el CPR de Avilés. Bajo el título "La historia de las mujeres y su inclusión en la enseñanza secundaria" tratábamos de aproximarnos a este campo de estudio, desde una perspectiva temática y diacrónica. Abordamos cinco grandes temas: el poder, la religiosidad, los saberes, los espacios y la distribución de la riqueza. Aunque el enfoque resultaba novedoso, al finalizar esta primera experiencia concluimos que resultaba de mayor provecho, si queríamos tener una incidencia real en las aulas, ofrecer una aproximación más cercana al currículo vigente, a la cronología en un sentido clásico y a los temas abordados en los centros educativos. Así, en 2013, y en el CPR de Oviedo, iniciamos una nueva serie de cursos que se estructuran por los marcos cronológicos convencionales. El primero de ellos abordó "Un enfoque coeducativo para la didáctica de la Historia: Prehistoria, Arqueología y Mundo Antiguo" y en 2014, nos centramos en la época contemporánea, la que tiene un mayor peso en el currículo de secundaria, y planteamos como título: "A la luz del género: nuevas perspectivas para la didáctica de la historia contemporánea".

Los objetivos perseguidos con esta oferta formativa pueden sintetizarse como sigue: 
- Ser capaces de analizar con espíritu crítico el currículo de las Ciencias Sociales vigente en la Educación Primaria y Secundaria Obligatoria en el Principado de Asturias y los materiales didácticos utilizados habitualmente en las aulas.

- Conocer los orígenes, la evolución y las perspectivas actuales de la Historia de las Mujeres.

- Familiarizarse con algunos de los temas más relevantes de la historia de las mujeres y del género y valorar su traslado a los centros educativos.

- Revistar algunos aspectos de la historia de España y Asturias desde la perspectiva de género.

- Valorar el patrimonio histórico existente en el Principado de Asturias y saber interpretarlo desde una perspectiva que incluya a mujeres y hombres.

- Tomar conciencia de la importancia de transmitir en los niveles básicos y medios de la educación una historia equilibrada con respecto a los sexos y que visibilice la presencia y el protagonismo de las mujeres en la historia.

- Valorar la relevancia de una enseñanza-aprendizaje de la historia que incluya el pasado en femenino para favorecer la igualdad entre mujeres y varones.

- Realizar propuestas prácticas para la inclusión de la historia de las mujeres en las aulas.

- Tomar contacto con nuevas fuentes y novedosas formar de leer las tradicionales para visibilizar el pasado en femenino.

Los resultados de estas experiencias han sido altamente satisfactorios. Uno de los logros más importantes radica en haber formado un grupo informal de profesoras y profesores comprometidos con la labor docente y la innovación educativa, interesados por la historia de las mujeres y las novedades historiográficas, que demandan nuevos cursos y que van poniendo en práctica en sus aulas estrategias y actividades para incluir una "historia mixta" en su trabajo como enseñantes. Esperamos mantener esta apuesta y, en colaboración con los CPRs, a partir de estas redes entre la investigación universitaria y la docencia en 
los institutos y colegios del Principado de Asturias -dado que también asiste profesorado de primaria-, generar grupos de trabajo que canalicen actividades, materiales y propuestas concretas para la modificación de las pautas tradicionales más negativas en la enseñanza de la historia y para paliar, en la medida de lo posible, los déficits actuales de la legislación educativa y los currículos vigentes.

\section{Conclusiones}

Cuando se hizo público el manifiesto de Historia a Debate, en 2001, el contexto internacional y la atención de los medios de información pasaba por el movimiento antiglobalización. Hoy, totalmente inmersos ya en ese proceso de mundialización y en la crisis económica y social vinculada al mismo, más que nunca se desvirtúa el papel de la historia que investigamos y enseñamos que parece destinada a convertirse en un retiro ocioso en lugar de constituir un espacio crítico para la reflexión y la conciencia social y ciudadana. Los desafíos de esta comunidad global pasan estos días por la búsqueda de soluciones a conflictos enquistados las atrocidades cometidas en la franja de Gaza-, al que se suma la dramática situación en el norte de Iraq y Siria ante el avance de ISIS. La problemática del cambio climático y sus efectos en las poblaciones, la persistencia de las desigualdades sociales, raciales y de género, obligan a la comunidad de historiadores e historiadoras a mantener un alto compromiso social y crítico. Desde esta perspectiva, las propuestas para el nuevo paradigma educativo de la historia, capaces de hacer frente a las tendencias en boga asociadas al aprendizaje "por competencias", alentadas desde instancias europeas y acogidas calurosamente en España, continúan siendo necesarias.

Desde nuestra posición, vinculada a la investigación en historia de las mujeres y comprometida con la enseñanza de una historia social, crítica, mixta y global, debemos poner la atención sobre las omisiones y los "olvidos conscientes" de la administración educativa en los currículos de primaria y secundaria que entrarán en vigor en virtud de la LOMCE en los próximos años. ${ }^{26} \quad$ Ante este

26 El calendario de aplicación de la LOMCE se inicia en el próximo curso 2014-2015 en primaria y en el curso 2015-2016 en secundaria. 
panorama poco alentador, pensamos que la formación continua puede ser una necesidad, a la par que una oportunidad, que debe incentivarse para en alguna medida poder vehicular hacia las aulas un aprendizaje en donde la historia de mujeres y hombres esté presente, y donde la innovación educativa desde una visión crítica con el pasado y con el presente cobre sentido.

\section{Bibliografía}

BERBEK SÁNCHEZ, S.; JIMÉNEZ; M. C.; MOSQUERA, N. P. Ideas que cambiaron el mundo. Madrid: Cátedra, col. Feminismos, 2013.

BORDERÍAS, C. (Ed.). Joan Scott y las políticas de la historia. Barcelona: Icaria. 2006.

BUENO DOMÍNGUEZ, M. L.: Reflexiones entre a la historia de las mujeres. ¿Una historia coyuntural? In: BARROS C. (Ed.). Historia a debate: actas del Congreso Internacional "A Historia a debate". Santiago de Compostela, p. 285-298. 1995.

BURGOS ALONSO, M.; MUÑOZ-DELGADO, M. C. Ciencias Sociales. Geografía e Historia, Madrid. 2008

CORRALES BERNABÉ, D. Estilos de aprendizaje de las competencias profesionales a lo largo de la vida. Estudio cualitativo en Cantabria. In: LÓPEZ, F. G.; GARCÍA-RUIZ, R.; FERNÁNDEZ, N. G.; ARELLANO, P. R.; ZUBIZARRETA, A. C. (Coord.). Estilos de aprendizaje: investigaciones y experiencias. $V$ Congreso Mundial de Estilos de Aprendizaje. Santander, Universidad de Cantabria. 2012. Disponible en: http://dialnet.unirioja.es/servlet/articulo?codigo $=4675074$ [Consultado el 21 de agosto de 2014].

FEMENÍAS, M. L. El feminismo postcolonial y sus límites. In: AMORÓS, C.; DE MIGUEL, A, (Ed.). Teoría feminista: de la Ilustración a la globalización. Vol. 3. Minerva, Madrid, 2003, p. 153-214.

GRAU COMPANY, S.; GÓMEZ LUCAS, C.; PERANDONES, G. T. M. La formación del profesorado como factor decisivo de la excelencia formativa Disponible en: http://rua.ua.es/dspace/bitstream/10045/13199/1/PROPUESTAS\%20CAP.\%201. pdf. [Consultado el 21 de agosto de 2014]

GRENCE RUIZ, T. (Dir.). Ciencias Sociales. Geografía e Historia. Madrid, Santillana. 2008.

DEL VAL VALDIVIESO, M. I. (Coord.). Historia de las mujeres: una revisión historiográfica. Universidad de Valladolid: Valladolid. 2004. 
MAÑERU MÉNDEZ, A.; HERRÁEZ, E. R. Educación para la igualdad de oportunidades. Madrid: Ministerio de Educación. 1992.

MONTERO CURIEL, M. El proceso de Bolonia y las nuevas competencias. Tejuelo, 9, p. 19-37. 2010.

MORANT, I. El sexo de la historia. Ayer. n. 17, p. 29-66. 1995.

MORENO, A. El arquetipo viril protagonista de la historia. Ejercicios de lectura no androcéntrica. Barcelona: La Sal. 1986.

NASH, M. Dos décadas de historia de las mujeres en España: una reconsideración. Historia Social, n. 9, p. 137-161. 1991

RAMOS PALOMO, M. D. Arquitectura del conocimiento, historia de las mujeres, historia contemporánea. Una mirada española. 1990-2005. Cuadernos de Historia Contemporánea. v. 28, p. 17-40. 2006. . ¿Clío en la encrucijada? A propósito de la historia de las mujeres (19902000). Arenal. n. 10, v.1, p. 81-103. 2013.

GONZÁlEZ URRUELA, E.; GONZÁLEZ, J. M. de J.; VALCÁRCEL, J. O. España. Geografía e Historia. Burgos: Santiago Rodríguez Hermanos. 1978.

ROGERO ANAYA, J. Movimientos de renovación pedagógica y profesionalización docente. Revista Latinoamericana de Educación Inclusiva, v.4, n.1, p. 141-166. 2010. Disponible en: http://www.rinace.net/rlei/numeros/vol4-num1/art7.pdf [Consultada el 12-8-2014].

SEBASTIÁN RAMOS, A. LIÉVANO, B. M.; SÁNCHEZ GARCÍA, M. F. Educar y orientar para la igualdad en razón de género. Madrid: Universidad Nacional de Educación a Distancia. 2001.

SEGURA GRAÍÑO, C. La incidencia de la mujer en la ciencia histórica. In: DURÁN, M. Á. (Ed.). Liberación y utopía. Madrid: Akal. p. 103-117. 1982.

\section{Documentos consultados disponibles on-line:}

Manifiesto de Historia a Debate. Pasado y Presente. Revista de Historia, v.8, núm.15, p.168-183. Disponible en:

http://www.ub.edu/histodidactica/images/documentos/pdf/manifiesto historia d ebate.pdf [Consultada el 21 de agosto de 2014]

Propuestas para el nuevo paradigma educativo de la historia. 2007. Disponible en: www.h- 
debate.com/cbarros/spanish/articulos/nuevo paradigma/npeducativo.htm [Consultada el 22 de agosto de 2014]

Declaración de Bolonia. Declaración conjunta de los Ministros Europeos de Educación. Bolonia, 19 de junio de 1999. Disponible en:

http://www.eees.es/es/documentacion [Consultada el 20 de agosto de 2014].

Declaración de Praga. Hacia el Área de la Educación Superior Europea.

Declaración del encuentro de los Ministros Europeos en funciones de la Educación Superior en Praga, 19 de mayo de 2001". Disponible en:

http://www.eees.es/es/documentacion [Consultada el 20 de agosto de 2014].

LOGSE. Ley 1/1990 de 3 de octubre (BOE de 4 de octubre de 1990). Disponible en: http://www.educacion.gob.es/mecd/oposiciones/files/logse.pdf [Consultado el 21 de agosto de 2014].

Ley Orgánica de 2/2006, de 3 de mayo, de Educación. Disponible en: http://noticias.juridicas.com/base datos/Admin/lo2-2006.html [Consultada el 20 de agosto de 2014].

Real Decreto 1631/2006, de 29 de diciembre, por el que se establecen las enseñanzas mínimas correspondientes a la Educación Secundaria Obligatoria. Disponible en: http://www.boe.es/boe/dias/2007/01/05/pdfs/A00677-00773.pdf [Consultada en 20 de agosto de 2014].

Decreto 74/2007, de 14 de junio, por el que se regula la ordenación y establece el currículo de la Educación secundaria obligatoria en el Principado de Asturias. Disponible en:

http://www.educastur.es/index.php?option=com content\&task=view\&id=997\&It emid=152 [Consultado el 27 de octubre de 2010].

Decreto 75/2008, 6 de agosto, por el que se establece la ordenación y el currículo del Bachillerato. Disponible en:

http://www.educastur.es/index.php?option=com content\&task=view\&id=2060\&I temid $=54$ [Consultado el 27 de octubre de 2010].

Proyecto de Real Decreto por el que se establece el currículo básico de la educación primaria, de la educación secundaria obligatoria y del Bachillerato conforme a la LOMCE. ". Disponible en: http://www.mecd.gob.es/servicios-alciudadano-mecd/dms/mecd/servicios-al-ciudadano-mecd/participacionpublica/cerrados/2013/curriculo-basico/RD-curr-culo-LOMCE-PR-ESO-BACH-V110-12-13.pdf [Consultada el 20 de agosto de 2014].

Real Decreto 126/2014, de 28 de febrero, por el que se establece el currículo básico de la Educación Primaria. Disponible en:

http://www.boe.es/boe/dias/2014/03/01/pdfs/BOE-A-2014-2222.pdf

[Consultada el 20 de agosto de 2014].

Mujeres y hombres en Asturias 2014". Disponible en:

http://institutoasturianodelamujer.com/iam/wp- 
content/uploads/2010/02/Diptico-Mujeres-y-hombres-en-Asturias-2014.pdf [Consultada el 20 de agosto de 2014].

Recebido em 23 de março de 2015.

Aprovado em 10 de junho de 2015. 